
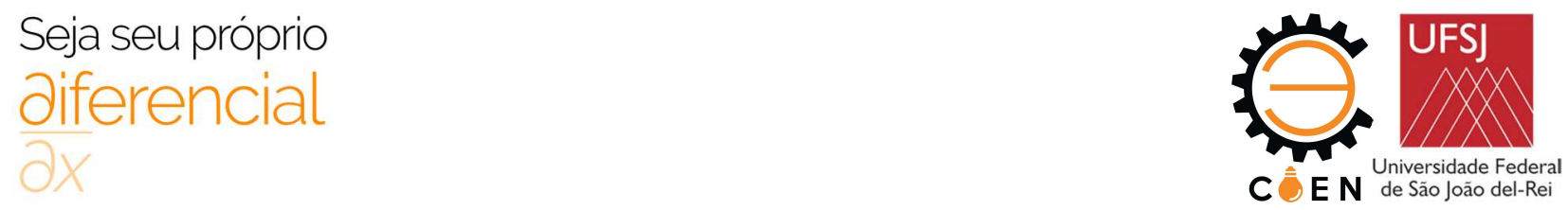

\title{
CONTROLE FUZZY PARA NAVEGAÇÃO AUTÔNOMA EM AMBIENTE DESCONHECIDO SUJEITO A MUDANÇAS
}

\author{
Larissa de Sousa Pinto ${ }^{(1)}$ (larissap@ita.br), Thaís da Costa Dias ${ }^{(2)}$ (thaisdias.engmec@hotmail.com), \\ Jorge Nei Brito ${ }^{(3)}$ (brito@ufsj.edu.br)
}

(1) Instituto Tecnológico de Aeronáutica (ITA) - Departamento de Eletrônica - Av. do Aeroporto $s \ln ^{\circ}$

(2) Universidade Federal de São João del-Rei (UFSJ) - PPMEC - Praça Frei Orlando, 170 - Centro, São João del-Rei - MG.

(3) Universidade Federal de São João del-Rei (UFSJ) - DEMEC - Praça Frei Orlando, 170 - Centro, São João del-Rei - MG.

RESUMO: Inteligência Artificial aplicada à navegação autônoma é uma área de grande interesse de pesquisa com vários desafios ainda a serem explorados. Pesquisas podem se beneficiar de ambientes desenvolvidos em plataformas de simulação e jogos digitais que proporcionam uma situação de teste próximas as da realidade fisica num ambiente sem custos, riscos e de fácil implementação. No contexto de Inteligência Artificial para resolução de problemas de navegação, a lógica fuzzy apresenta uma forma de manuseio de informações capaz de conectar o conhecimento humano com a forma quantitativa de processamento de dados de computadores. Este trabalho apresenta fundamentação e conceitos necessários para a implementação de um sistema de controle para um veículo autônomo capaz de percorrer pistas de variados formatos via simulação na plataforma TORCS. Essa plataforma de código aberto oferece um elaborado sistema que considera aspectos físicos importantes como colisões, tração, aerodinâmica e consumo de combustivel. $O$ controle inteligente do sistema utiliza lógica fuzzy e é responsável por encontrar ações de controle para o volante, acelerador, freio e marcha do veiculo a fim de gastar o menor tempo em uma volta.

PALAVRAS-CHAVE: Controle Fuzzy, Navegação Autônoma, TORCS, Inteligência Artificial em Jogos.

\section{INTRODUÇÃ̃o}

O desenvolvimento de métodos e tecnologias aplicadas à robótica móvel e sistemas autônomos tem despertado o interesse de pesquisadores do meio acadêmico e industrial em vista da diversidade de possíveis aplicações. A pilotagem de carros é uma tarefa reconhecidamente difícil. Especialistas nessa atividade necessitam realizar diversas sequencias de testes e ações para que o veículo atinja seu limite de desempenho. Diante deste cenário, o uso de métodos de Inteligência Artificial (IA) é interessante para o desenvolvimento de modelos e controladores e, consequentemente, para a automatização da pilotagem.

A construção ou uso de veículos reais apropriados para testes de métodos inteligentes é um processo oneroso e demorado, que envolve grande demanda de dispositivos e materiais. Os procedimentos de teste geralmente têm alto custo e envolvem risco. Em contrapartida, diversas plataformas de jogos de corrida se mostram cada vez mais capazes de simular com detalhes o mundo real e a dinâmica de veículos. Com isso, o uso de jogos eletrônicos e o desenvolvimento de técnicas de inteligência computacional se mostram como um domínio promissor a ser explorado no contexto de navegação autônoma.

Neste cenário de jogos e simuladores existentes, o TORCS (The Open Racing Car Simulator) se mostra como um interessante ambiente virtual para pesquisa e desenvolvimento científico. Ele é tido como plataforma de testes, pois implementa um ambiente realista considerando conceitos físicos aliado a uma arquitetura modular que permite a construção e integração de modelos e controladores.

Desenvolver veículos que não dependem de motorista não é uma atividade de pesquisa recente. A literatura sobre o tema apresenta diversas aplicações bem-sucedidas. Em ambientes reais, podemos citar as pesquisas realizadas na Universidade de Carnegie Mellon, pioneira nesse segmento 


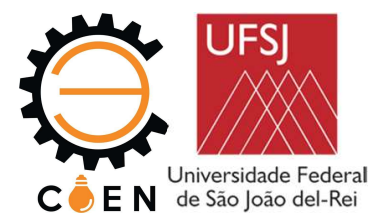

com os projetos Navlab1 e ALV em meados da década de 80, bem como os projetos preparados pelo EUREKA Prometheus Project, que em 1994 conseguiram conduzir um veículo autônomo por mais de $1000 \mathrm{~km}$ no trânsito normal de uma rodovia em Paris. Quanto a ambientes de simulação de jogos de corrida, podemos citar alguns candidatos em campeonatos internacionais, como o SCRC, como por exemplo o projeto AUTOPIA, que utiliza uma arquitetura modular, técnicas fuzzy e algoritmos genéticos. Outros controladores competitivos que apresentam bons resultados podem ser citados, como o Mr. Race e o COBOSTAR.

No presente trabalho, estudamos o desenvolvimento e a implementação de um controlador fuzzy tipo Mamdani para um veículo autônomo. O controlador fuzzy foi testado em diferentes pistas do simulador TORCS. Em particular, o foco é no controle de ângulo do volante, aceleração e freio. Os parâmetros do controlador foram ajustados a partir de conhecimento especialista sobre o domínio da aplicação.

\section{OBJETIVOS}

O objetivo deste trabalho é, baseado numa arquitetura de controle reativa, desenvolver algoritmos de controle utilizando lógica fuzzy, para garantir que um veículo, simulado na plataforma de jogos TORCS, seja capaz de realizar manobras a fim de alcançar um ponto objetivo no menor tempo possível e em diferentes pistas disponíveis no ambiente de simulação. O controlador fuzzy atua na direção, aceleração e freio do veículo a partir de medições do ambiente em torno.

\section{REFERENCIAL TEÓRICO}

Os principais blocos constituintes de um piloto automático são:

Navegação: permite o conhecimento dos estados do veículo, posição, velocidade e aceleração, em relação a um determinado referencial, ou seja, consiste em determinar (com acurácia) a posição e a velocidade de um corpo em relação a uma referência conhecida.

- Guiagem: tem o objetivo de determinar a melhor trajetória e os movimentos para executá-la.

- Controle: Tem a função de realizar o controle do veículo segundo os ângulos associados aos eixos: Ox, Ou. De forma geral, estes blocos estão presentes em qualquer veículo autônomo, sendo os conceitos envolvidos os mesmos para veículos aéreos, marítimos ou terrestres.

\subsection{Navegação autônoma}

No sentido geral, um Unmanned Ground Vehicle (UGV), ou Veículo Terrestre não tripulado, é qualquer peça de equipamento mecanizado que se move sobre uma superfície e serve como meio de carregar ou transportar algo, não necessariamente um ser humano (GAGE, D. W., 1995).

Veículos autônomos são, portanto, sistemas móveis capazes de receber informações e a partir delas tomar decisões por conta própria a fim de executar tarefas programadas. Os sistemas autônomos geralmente possuem os seguintes elementos ou características (PEREIRA, F., 2005):

- Sensores úteis para obter dados do ambiente;

- Controle e Inteligência, ou seja, como o robô transforma suas percepções em ações a serem executadas;

- Capacidade de processamento para interpretar os dados do ambiente e tomar decisões;

- Sistema de comunicação, útil para obter informação complementar àquelas provenientes de sensores e interagir com um operador humano, eventualmente recebendo sua intervenção;

IX COEN - Congresso de Engenharias da UFSJ Interconexão. 

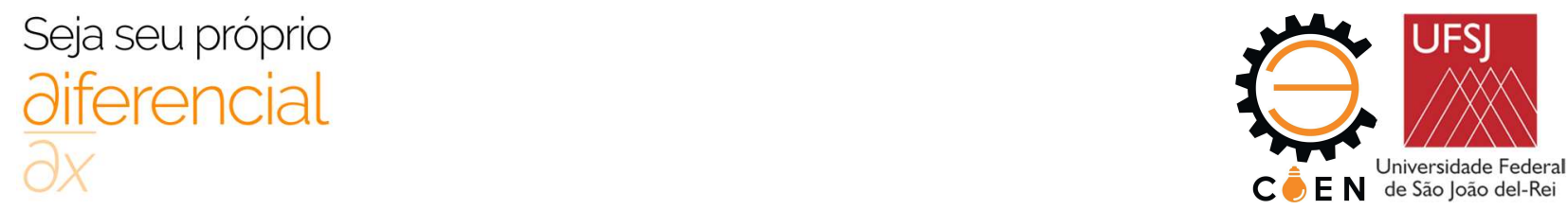

- Mobilidade e adaptação à ambientes variáveis.

A navegação autônoma de veículos terrestres tem recebido bastante atenção e esforços de desenvolvimento e aprimoração desde 1960, inicialmente em aplicações militares e de exploração. Posteriormente robôs móveis ganham novas aplicações em atividades industriais, domésticas, exploração de ambientes de difícil acesso, entre outras (CHOSET et. al, 2005); (SECCHI, 2008). Atualmente os estudos sobre navegação autônoma estão sendo impulsionados principalmente em quatro áreas:

- Exploração espacial em expedições em outros planetas e corpos celestes como o jipe-robô Curiosity, enviado para explorar o planeta Marte pela Agência Espacial Americana, NASA, em 2012;

- Aplicações militares, como por exemplo as operações da DARPA (Defense Advanced Research Projects), agência norte-americana responsável por desenvolvimento tecnológico e inovações na área militar. A DARPA tem como um de seus objetivos a transformação de um terço da frota militar dos EUA em veículos autônomos capazes de atuar inclusive em áreas altamente povoadas;

- Robótica móvel utilizada na agricultura, no ramo da chamada "agricultura de precisão", especialmente em aplicações para aquisição de dados no campo, coleta de frutos, plantio e tratamento da terra;

- E. por último, em Sistemas de Auxílio ao Condutor (SAC), que traz ao mercado consumidor produtos mais seguros, confortáveis e inclusivos.

\subsection{Navegação de UGV baseada em sensores}

Em navegação autônoma é necessário que o robô adquirira informações para que decisões sejam tomadas. Da mesma forma, humanos tomamos decisões a partir dos estímulos que chegam aos seus órgãos sensoriais. Podemos dizer que robôs precisam "sentir" o ambiente no qual atua, e isso é feito através da leitura e interpretação dos dados de seus sensores. Estes podem ser sensores de posição, orientação, contato, deslocamento e velocidade, e de obstáculos, e.g., sensores infravermelhos, ultrassônicos, encoders, câmeras etc.

Qualquer sistema de percepção possui limitações; ele pode ser parcial e incompleto, ou seja, não apresenta todas as informações sobre o ambiente de atuação, são ruidosos e sujeito a erros; informações irrelevantes podem atrapalhar a observação de dados importantes. Essas limitações acarretam a existência de diversos erros no o sistema, por isso é importante a realização de procedimentos básicos como calibração e manutenção, visto que a percepção é uma das principais rotinas dos sistemas autônomos, agregando um maior nível de autonomia e robustez a um robô. Os sensores de diferentes tipos podem servir para se obter um sistema do tipo realimentado, tornando processos controláveis. Isso é necessário visto que na prática a execução de um comando normalmente não possui como resultado uma operação perfeita dos atuadores, devido a características mecânicas dos próprios atuadores e do ambiente de ação do sistema. Um exemplo, é o uso de encoders (sensores eletromecânicos, utilizados para conversão de movimentos rotativos ou lineares em impulsos elétricos) que permite a medição real do deslocamento das rodas de um veículo (CARVALHO JR., 2007).

O método reativo é baseado no princípio de ação e reação. Basicamente, através de dados obtidos de sensores, um robô reconhece os obstáculos e reage de modo a evitá-los. O controle reativo é normalmente o mais simples de ser implementado (reativo puro). Ele utiliza, em comparação a

IX COEN - Congresso de Engenharias da UFSJ Interconexão. 

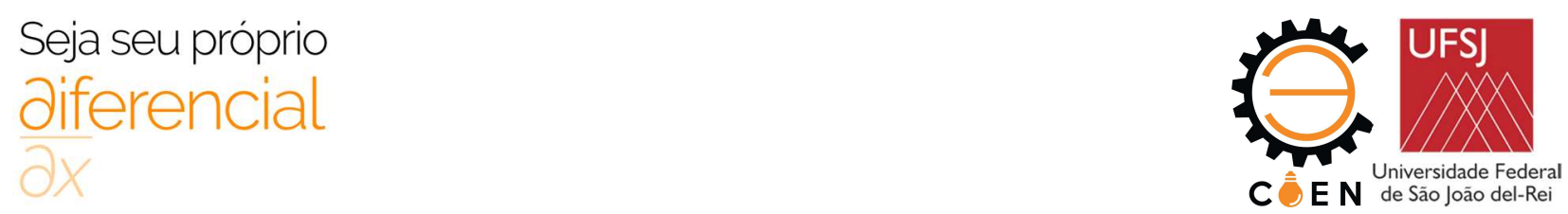

outros controles, menos recurso computacional. De um modo geral, a rotina é: ler sensores; processar os dados obtidos; levantar a resposta a partir da análise dos dados; e enviar comando para atuadores. Um sistema reativo é útil para rotinas como: desviar de obstáculos e seguir um objeto, por exemplo (CARVALHO Jr. H. H., 2007); (PEREIRA, A.H. et. al., 2011).

O controle deliberativo (ou cognitivo) baseia-se em uma sequência de ações, estabelecidas previamente à execução, utilizando conhecimentos que o sistema possui sobre o ambiente de ação, e.g., mapa do ambiente, rotas, GPS. Esse controle, exige maior nível de raciocínio e tomadas de decisões, sendo mais complexo de ser implementado. O controle "deliberativo puro" possui limitações em situações como, por exemplo, em casos de ocorrência de obstáculos móveis obstruindo sua rota (HEINEN, F. J., 2000). Percebe-se que ambos os controles, reativo e cognitivo, possuem pontos positivos e negativos. Pode-se dizer que o ideal em um sistema seria a utilização das duas técnicas de controle, combinando a capacidade de reação de um sistema reativo com a capacidade de planejamento

e execução de um sistema cognitivo. Desta combinação reativo-cognitivo, surgem os sistemas hierárquicos ou híbridos.

Em (PEREIRA, C. et. al., 2011) é proposto o uso da plataforma Robotino da Festo que possui nove sensores do tipo infravermelhos, distribuídos ao redor do robô, onde foram considerados apenas cinco deles para a construção de um modelo fuzzy que garantia um monitoramento em 160 graus, trabalhando apenas com pares de sensores e excluindo-se o desvio de objetos pequenos. Já em (CARVALHO JR., 2007), todo o controle é feito a partir do monitoramento do ambiente realizado através do processamento de imagens geradas a partir de uma câmera localizada acima da área de teste.

Em (RAMOS, G., 2014), o sistema proposto realiza a fusão de vários sensores, sendo composto por GPS (Sistema de Posicionamento Global), DGPS (Sistema de Posicionamento Global Diferencial) e uma plataforma INS (Sistema de navegação Inercial), uma plataforma constituída por sensores do tipo girômetros, acelerômetros e bússolas magnéticas.

\subsection{Controle fuzzy}

Os sistemas Fuzzy, também conhecidos como sistemas nebulosos ou difusos, é um sistema matemático capaz de modelar a informações imprecisas presentes na linguagem natural, sendo constituídos de quatro elementos, (1) módulo de fuzzificação (2) módulo base de regras (3) módulo de inferência e (4) módulo de defuzzificação, que serão descritos a seguir.

As técnicas dos conjuntos fuzzy atualmente têm alcançado diversas áreas de aplicação sendo o uso delas no controle fuzzy, uma área que tem possibilitado a automação de diversos processos. Estes processos vão desde tarefas simples até o controle de sofisticados sistemas e processos industriais. Por isso, em diversas áreas do conhecimento, muitos pesquisadores vêm desenvolvendo algoritmos específicos utilizando esta teoria para a análises e simulações.

A lógica fuzzy traz uma nova forma de trabalhar informações imprecisas, levando expressões verbais qualitativas, imprecisas, vagas e do cotidiano dos humanos para o sistema numérico, fazendo então uma conexão sistemática entre a linguagem e o pensamento humano com a linguagem do computador. Pode se dizer então que essa técnica incorpora a forma humana de pensar em um sistema de controle. Assim um controlador fuzzy pode ser projetado para comportar-se conforme o raciocínio dedutivo, isto é, o processo que as pessoas utilizam para inferir conclusões baseadas em informações que já conhecem, sendo assim, é capaz de capturar o conhecimento do operador humano sobre 
características de sistemas não lineares e implementá-lo em um controlador computacional com desempenho relativo ao humano.

O uso das regras fuzzy confere ao sistema de controle diversas vantagens como: simplificação do modelo do processo; melhor tratamento das imprecisões causadas pelos sensores utilizados; facilidade na implementação das regras de controle; atendimento a múltiplos objetivos de controle; e incorporação do conhecimento de especialistas humanos. Porém, tanto as entradas do sistema (leituras de sensores) quanto as saídas (sinais esperados pelos atuadores do sistema de controle) não pertencem a linguagem desta lógica, fazendo-se necessário a conversão entre a lógica aritmética e a lógica fuzzy (FERNANDES et al., 2005). A Figura 1 mostra o diagrama de blocos de um sistema fuzzy aplicado no controle de um processo físico.

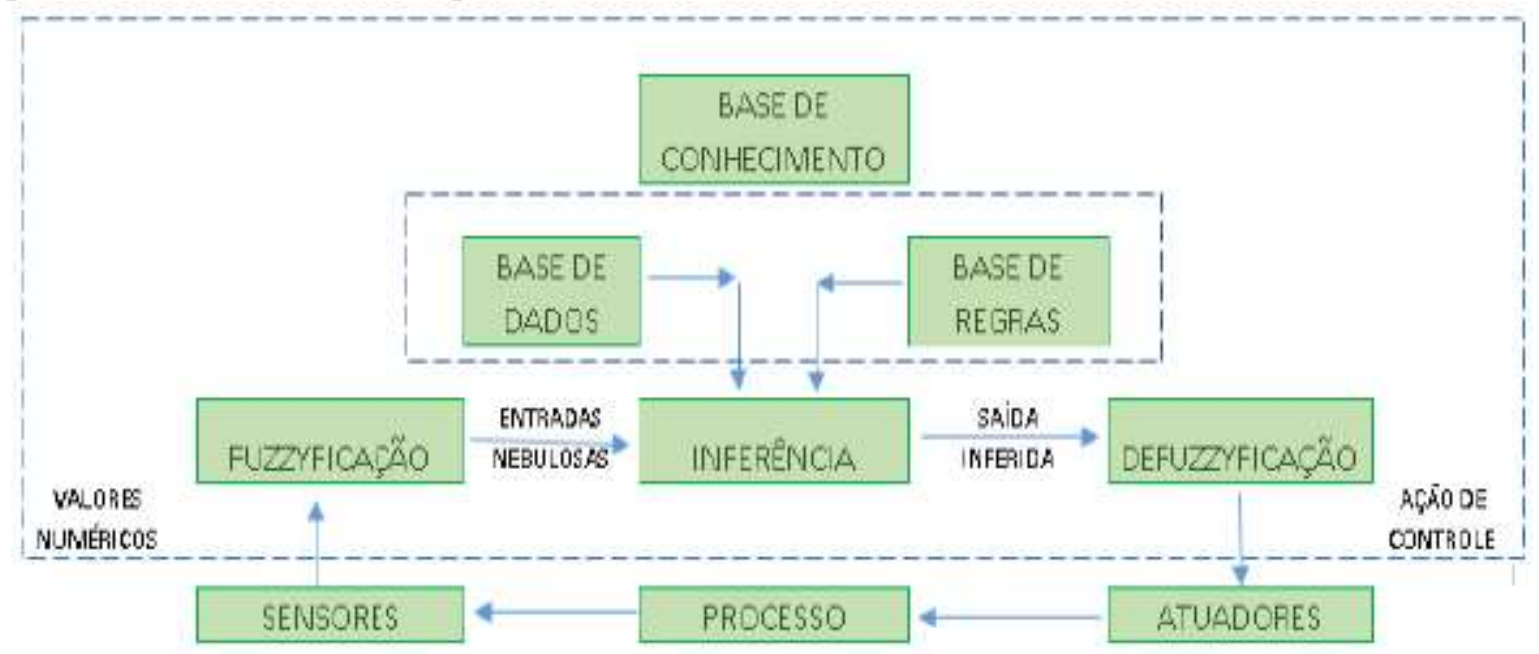

Figura 1. Diagrama de blocos de um sistema fuzzy.

A lógica clássica pode ser representada pela função indicadora $I($.$) , que assume somente os$ valores, $\{0,1\}$, onde 0 indica que o elemento não pertence, e 1 significa que o elemento pertence ao conjunto em questão. Sendo assim, dado um conjunto $A$ que pertence ao universo $U$, um elemento $x$ de $U$ pode assumir dois estados em relação ao conjunto $A$, que é representado pela função:

$I A(x): I A(x)=\{1$ se $x \in A$, ou 0 se $x \notin A\}$.

Na lógica fuzzy, um mesmo elemento pode assumir 3 estados. Considerando que $x \in \mathrm{U}$ temse:

- $\quad x$ pode pertencer integralmente ao conjunto $A$;

- $\quad x$ pode não pertencer a $\mathrm{A}$;

- $\quad x$ pode pertencer parcialmente ao conjunto $A$.

Como um elemento pode pertencer parcialmente a um conjunto é necessário trocar a função indicadora $\mathrm{I}()=.\{0,1\}$ por uma função pertinência $\mu()=.[0,1]$, isto é, o grau de pertinência de um elemento a um conjunto é um número real no intervalo fechado $[0,1]$ ao invés de apenas os dois valores do intervalo como na lógica clássica. Assim passamos a definir o conjunto fuzzy como:

$\mathrm{A}=\{\mathrm{x} \in \mathrm{U} \mid \mu \mathrm{A}(\mathrm{x})=\xi$, onde $0 \leq \xi \leq 1\}$.

IX COEN - Congresso de Engenharias da UFSJ Interconexão. 

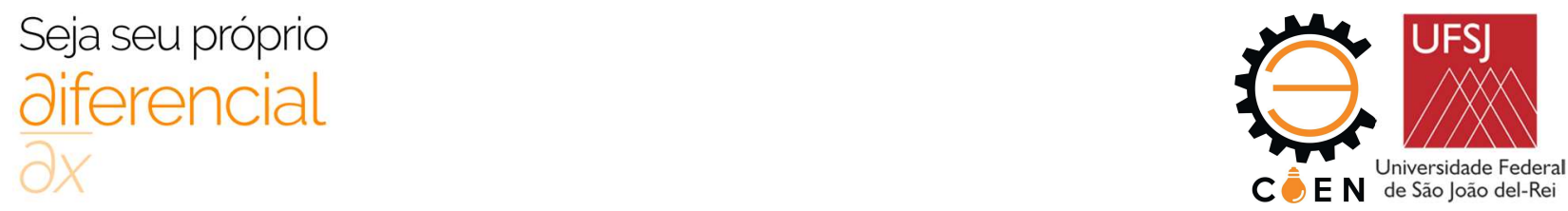

Podemos dizer que um sistema baseado em lógica fuzzy pode ter sua estrutura esquematizada pelos seguintes elementos: fuzzificador, máquina de inferência ou lógica de tomada de decisões, e, por fim, o elemento defuzzificador.

- O processo de fuzzificação consiste em obter a partir de uma variável numérica $\mathrm{x} \in \mathrm{U}$, o seu grau de pertinência aos conjuntos Ai contidos no universo U.

- O processo de inferência realiza as operações com conjuntos fuzzy. Podemos usar as respectivas definições para os operadores $\mathrm{A} \cup \mathrm{B}, \mathrm{A} \cap \mathrm{B}, \neg \mathrm{A}$ :

$$
\begin{gathered}
\mathrm{A} \cup \mathrm{B}=\max (\mu \mathrm{A}, \mu \mathrm{B}) \\
\mathrm{A} \cap \mathrm{B}=\min (\mu \mathrm{A}, \mu \mathrm{B}) \\
\neg \mathrm{A}=1-\mu \mathrm{A}
\end{gathered}
$$

Quanto a operação implica $(\Rightarrow)$ a operação leva o valor da função pertinência obtida do conjunto resultante do primeiro termo para o conjunto do segundo termo.

- O processo de defuzzificação consiste em obter a partir do valor da função pertinência resultante das diversas regras de inferência um valor numérico de saída.

Nesse trabalho usaremos o sistema de inferência do tipo Mandani. A máquina de inferência recebe valores fuzzy a partir do fuzzificador cuja responsabilidade é converter valores escalares para valores fuzzy equivalentes como explicado na seção anterior. Para tanto, é necessário que sejam definidas as funções de inclusão, de modo que os valores escalares sejam mapeados em valores no intervalo $[0,1] \in \mathrm{R}$. Em seguida, executa-se as regras existentes no banco de regras e gera-se um conjunto fuzzy de saída a partir da agregação de todas as regras disparadas. Por fim, o conjunto fuzzy obtido é enviado para o defuzzificador, que retorna um valor escalar para os atuadores.

Nesse procedimento a inferência fuzzy é então a avaliação das regras que relacionam as variáveis de entrada com a resposta do sistema. AND - é a interseção através de normas T e OR - é a união através de normas $\mathrm{S}$. Esse procedimento é feito em duas fases: avaliação da implicação de cada regra e a composição das conclusões de todas as regras em um valor final.

Segundo Rezende (2005), o método Mamdani foi por muito tempo um padrão e o modelo de inferência mais popular no uso dos conceitos da lógica fuzzy em sistemas de controle. É baseado numa estrutura simples de operações min-max, envolvendo regras de inferência do tipo: Se x é A e y é B Então z é C onde A, B e C são conjuntos fuzzy.

A regra utilizada para se realizar inferência no modelo de Mamdani é chamada de Max-Min. Nesse tipo de regra são utilizadas operações de união e interseção entre conjuntos, por meio dos operadores de máximo e mínimo, respectivamente, e que serão apresentados na próxima secção.

A implementação das regras é feita a partir da definição de operadores para o processamento do antecedente da regra e da função de implicação que vai definir seu consequente. A ação do controlador fuzzy é definida pela agregação das $\mathrm{n}$ regras que formam o algoritmo. Esta agregação resulta no conjunto fuzzy, que define a saída do controlador. A saída efetiva do controlador é então obtida por meio do processo de defuzzificação.

Funções de pertinência fuzzy representam os aspectos fundamentais de todas as ações teóricas e práticas de sistemas fuzzy. Uma função de pertinência é uma função numérica gráfica ou tabulada que atribui valores de pertinência fuzzy para valores discretos de uma variável, em seu universo de discurso. É preciso lembrar que o universo de discurso de uma variável representa o intervalo numérico de todos os possíveis valores reais que uma variável específica pode assumir. 


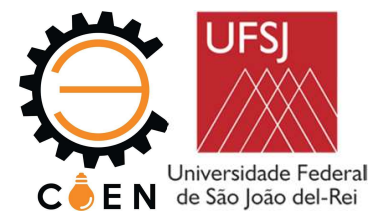

Na lógica Fuzzy a função de pertinência de intersecção $A \cap B$ pode ser implementada usando uma família de operadores conhecidos como t-norma. A t-norma é uma função de duas entradas definidas nos domínios t: $[0,1] \mathrm{X}[0,1]->[0,1]$, onde " $\mathrm{X}$ " é o operador de produto cartesiano. A noção de intersecção dos conjuntos está relacionada com o conectivo lógico "E". Este operador faz um mapeamento entre duas funções fuzzy ponto a ponto e deve satisfazer as seguintes condições e propriedades:

- Condições de contorno:

$$
\begin{aligned}
& \mathrm{xt} 0=0, \forall \mathrm{u} \in[0,1] \\
& \mathrm{xt} 1=\mathrm{x}, \forall \mathrm{u} \in[0,1]
\end{aligned}
$$

- Propriedade comutativa:

$$
\mathrm{xt} y=\mathrm{y} \mathbf{t} \mathrm{x}
$$

- Propriedade associativa:

$$
\mathrm{x} \mathrm{t}(\mathrm{y} \mathbf{t} \mathrm{z})=(\mathrm{x} \mathbf{t} \mathrm{z}) \mathbf{t} \mathrm{z}
$$

- Condições monotônicas para $\mathrm{x} \leq \mathrm{ye} \mathrm{w} \leq \mathrm{z}$, $\mathrm{x} \mathrm{t} \mathrm{w} \leq \mathrm{yt} \mathrm{z}$

As t-normas podem ser: intersecção, produto algébrico, produto logarítmico, produto inverso, produto limitado e produto drástico. Os mais utilizados são:

- Intersecção:

$$
\mathrm{xt} y=\min (\mathrm{x}, \mathrm{y})
$$

- Produto algébrico:

$$
\mathrm{xt} y=\mathrm{x} \mathrm{y}
$$

Já a função de pertinência de união $A \cup \mathrm{B}$ é implementada através dos operadores s-norma. Tendo, assim como no operador anterior, duas entradas nos domínios s: $[0,1] \mathrm{X}[0,1]->[0,1]$, satisfazendo as mesmas condições de contorno e monotônicas e as propriedades comutativa, associativa. A operação de união de dois conjuntos está associada ao conectivo lógico "OU". As snormas podem ser: união, soma algébrica, soma limitada, soma logarítmica, soma disjunta, e soma drástica. As mais utilizadas são:

- União:

- Soma algébrica:

$$
x \boldsymbol{s} y=\max (\mathrm{x}, \mathrm{y})
$$

$$
x \boldsymbol{s} y=x+y-x y
$$

O complemento de um conjunto fuzzy, seja $\mathrm{U}$ o universo de discurso, com $x \in \mathrm{E}, \mathrm{M}=[0,1]$; onde o conjunto A está contido em U. 0 complemento de $A$ em relação a $\mathrm{U}$, é denominado $A$ ', e é definido como o conjunto de todos os elementos $\mathrm{x} \in \mathrm{U}$ que não estão presentes em $\mathrm{A}$.

No modelo de Mamdani temos:

$$
\mathrm{T}(a, b)=\min (a, b), \mathrm{I}(\mathrm{a}, \mathrm{b})=\min (a, b) \text { e } \nabla(\mathrm{a}, \mathrm{b})=\max (\mathrm{a}, \mathrm{b})
$$

e no modelo de Larsen temos:

$$
\mathrm{T}(a, b)=a^{*} b, I(a, b)=\left(a^{*} b\right) \text { e } \nabla(a, b)=\max (a, b) .
$$




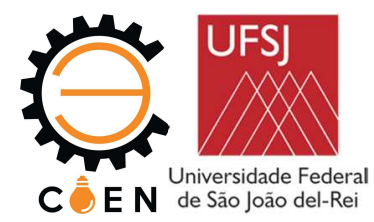

Após executadas a regras fuzzy, é necessário realizar a defuzzificação, nesse processo o valor da variável linguística de saída inferida pelas regras fuzzy será traduzido num valor discreto. O objetivo é obter-se um único valor numérico discreto que melhor represente os valores fuzzy inferidos da variável linguística de saída, ou seja a distribuição de possibilidades. Assim, a defuzzificação é uma transformação inversa que traduz a saída do domínio fuzzy para o domínio discreto. Apesar de não haver nenhum procedimento sistemático para a escolha da estratégia de defuzzificação, as mais comuns incluem: o critério do máximo (MAX), que escolhe o ponto onde a função inferida tem seu máximo, a média dos máximos (MDM), que representa o valor médio dentre todos pontos de máximo quando existe mais de um máximo, e o método do centro de área (CDA), que retorna o centro de área da função inferida.

O método de defuzzificação do centro da área, também chamado de centroide ou Centro-deGravidade é o método que utilizaremos neste trabalho, ele é semelhante à média ponderada para a distribuição de dados. Aqui, o centro de gravidade dá a média das áreas de todas as figuras que representam os graus de pertinência de um subconjunto fuzzy $(\mu O U T)$, fuzzy esse termo de saída fuzzy é composto pela união de todas as contribuições de regras. O centroide é um ponto que divide a área de $\mu O U T$ em duas partes iguais. Onde $\mu$ OUT(ui) é área de uma função de pertinência (como, por exemplo, "Reto" ou "Direita") modificada pelo resultado da interferência fuzzy (como, por exemplo, 0,2 ou 0,8 respectivamente), e ui é a posição do centroide da função de pertinência individual - "Reto" ou "PoucoDireita" respectivamente;

$$
u^{*}=\frac{\sum_{i=1}^{N} u i \mu \operatorname{OUT}(u i)}{\sum_{i=1}^{N} \mu \operatorname{OUT}(u i)}
$$

\section{MATERIAIS E MÉTODOS}

Neste capítulo será abordado em detalhes informações a respeito da construção do piloto automático construído. A primeira seção, informações a respeito do simulador escolhido (TORCS) e o modo de comunicação entre o simulador e o controlador implementado em MATLAB® serão apresentadas. Na segunda seção, será apresentado o esquema fuzzy de controle assim como os parâmetros utilizados para o controle dos atuadores do carro.

\subsection{Simulador TORCS}

O uso de ambientes virtuais de simulação é uma ferramenta que apresenta vantagens como economia de recursos e tempo, diminuição de acidentes e consequentemente danos ao robô e operadores, aperfeiçoamento de hardware e software visto que torna mais simples o teste, ajuste e avaliação de novas implementações.

O TORCS, “The Open Racing Car Simulator”, é uma aplicação de jogo e uma base de testes para controladores autônomos utilizada como plataforma de simulação em diversas competições entre métodos de IA em ambientes de corrida como a Simulated Car Racing Championship (SCR), campeonato este dividido em três etapas, cada uma ocorrendo em uma conferência internacional diferente (IEEE Congress on Evolutionary Computation, ACM Genetic and Evolutionary Computation Conference e IEEE Symposium on Computational Inteligence and Games) . Este simulador proporciona um ambiente customizável, gráficos $3 \mathrm{D}$, uma plataforma poderosa de simulações físicas levando em consideração aspectos importantes como colisões, tração, aerodinâmica e consumo de combustível, e fornece uma variedade de pistas, veículos e pilotos. 

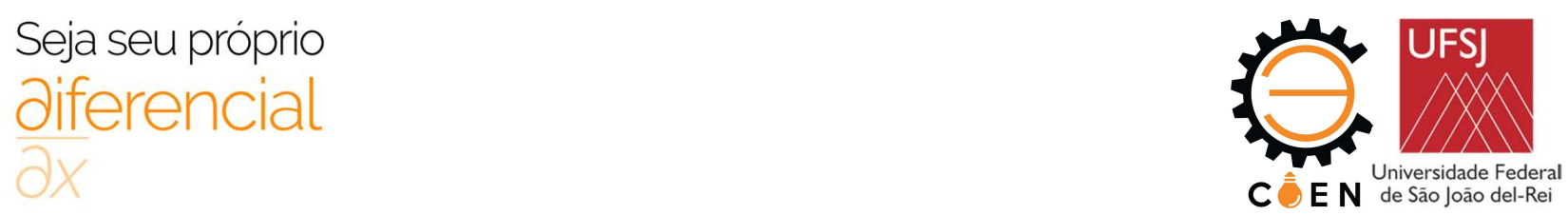

O programa é de código aberto e permite criação de novos controladores. Neste trabalho, utilizaremos uma arquitetura cliente-servidor, onde os controladores são executados em processos separados que se comunicam com o servidor através de uma conexão UDP. A simulação é feita em tempo real. O servidor envia os valores dos sensores para cada controlador e aguarda 10ms (tempo real) por algum comando a ser realizado. Caso não haja uma nova instrução, a simulação continua e a última ação é repetida. Como a plataforma TORCS é separada entre o código do driver e o próprio simulador, a escolha da linguagem de programação usada para implementar os controladores (bots) fica livre, à escolha do programador.

Os controladores conectados à arquitetura cliente-servidor recebem as informações organizadas, de forma que cada veículo capta um conjunto restrito de dados sobre o ambiente em sua volta e sobre o próprio controlador, com alcance limitado. Esta observabilidade parcial assemelha-se à situação de um robô real.

O motor do carro é controlado por meio de um conjunto de atuadores: o volante (Steer), a aceleração (accel), o pedal do freio (brake) e as engrenagens de velocidade (gear). O controlador recebe como informação de entrada o estado atual do carro e sua situação na pista. A partir desses dados calcula-se e decide-se as ações que devem ser tomadas para próximo passo da simulação como direção, mudança de marcha, aceleração ou freio e acionamento da embreagem.

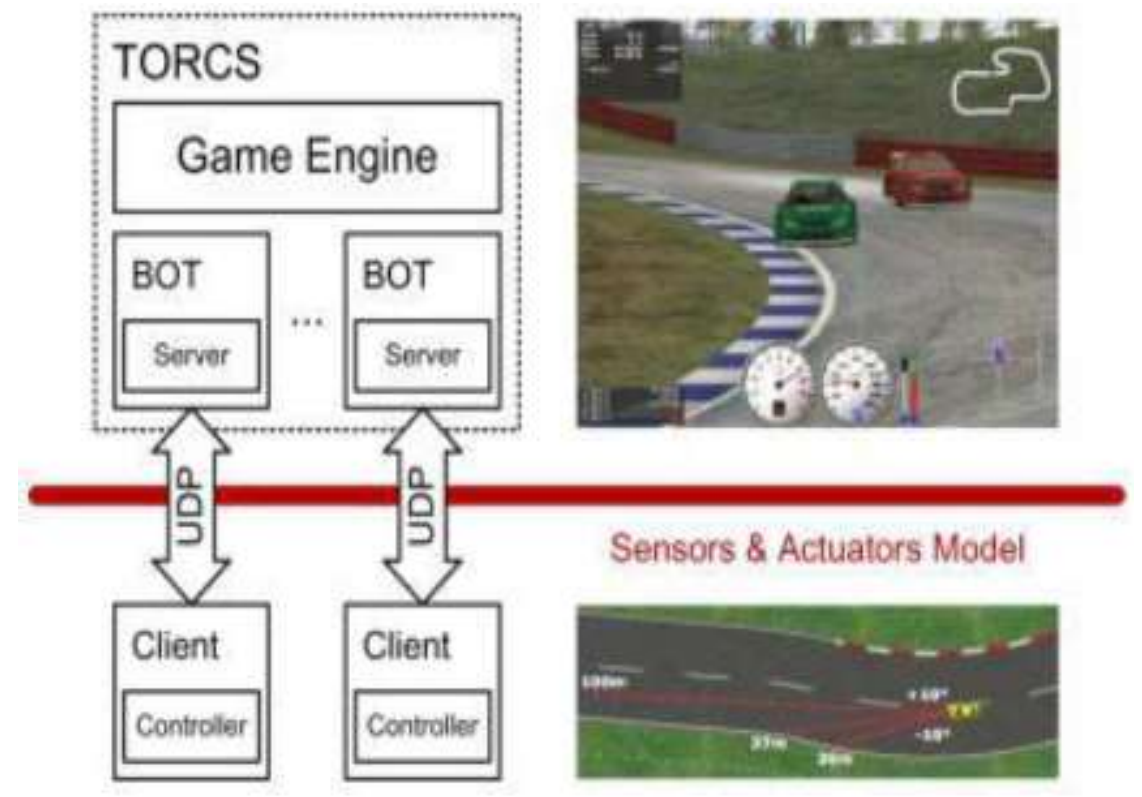

Figura 2. Arquitetura cliente-servidor do simulador TORCS. LOIACONO, D. (2013).

\subsection{Esquema de controle}

Foi implementado o seguinte sistema de o controle:

Dois controladores independentes, um para o freio e acelerador e outro apenas dedicado ao volante. O controle de marcha foi feito separadamente utilizando de estruturas de comparação simples como mostrado nos próximos tópicos.

O controle do carro será feito em três funções separadas mostradas a seguir, uma para o controle do volante, uma para a engrenagem e outra para acelerador e freio. 


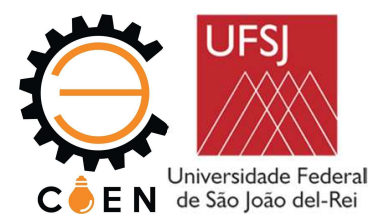

Para controlar o volante, o controlador chama a função calculeteSteer, fazendo a leitura dos seguintes sensores: sensors.track, sensors.opponents e sensors.trackPos. As Figuras 3, 4 e 5 a seguir mostram respectivamente os sensores track, que fornece um vetor com as distâncias da borda da pista a partir 19 sensores posicionados em diferentes ângulos partindo do centro do carro, trackPos, que informa a posição do veículo em relação à pista, e opponents, retorna a distância do adversário mais próximo em intervalos angulares, fundamentais para estimar a situação atual do veículo na pista.

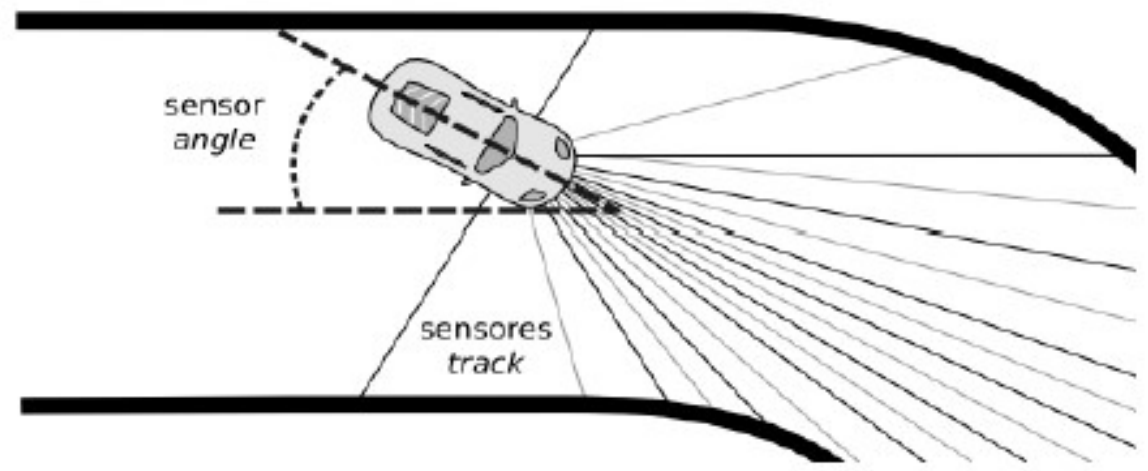

Figura 3. Esquema dos sensores angle que retorna o ângulo entre a pista e o veículo e sensor track que retorna a distância de 19 sensores entre o carro e a borda da pista.

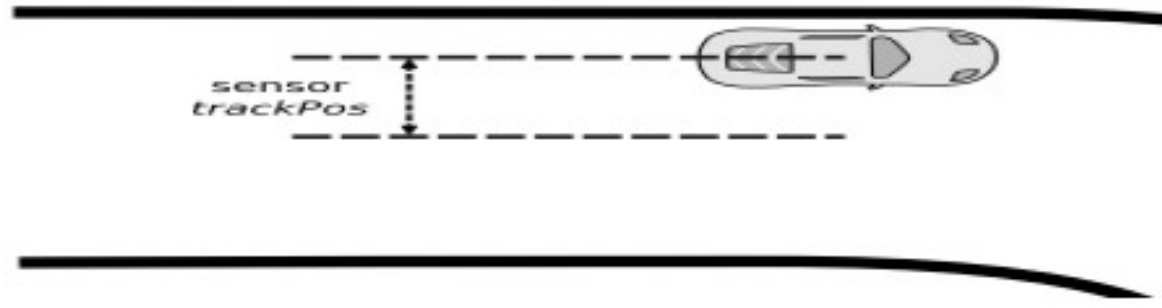

Figura 4. Esquema dos sensores angle que retorna o ângulo entre a pista e o veículo e sensor track que retorna a distância de 19 sensores entre o carro e a borda da pista.

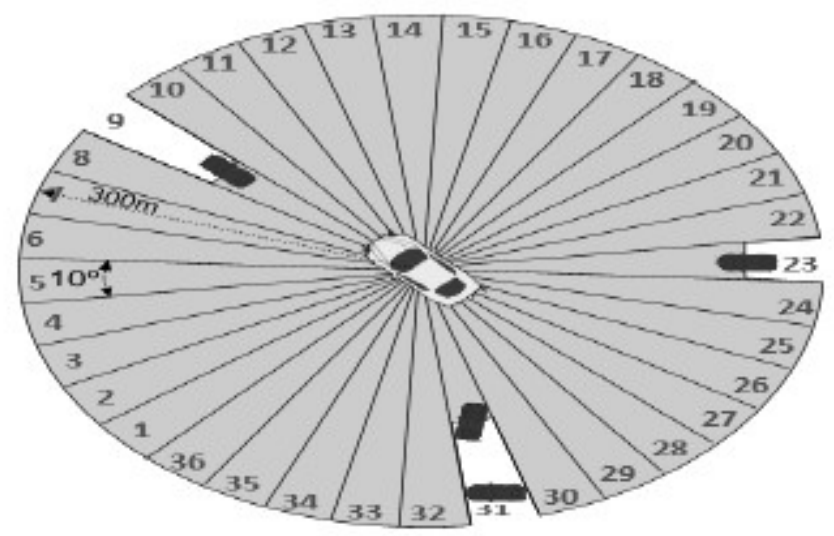

Figura 5. Esquema sensor opponents retorna um vetor com 36 elementos contendo a distância dos oponentes que estão a uma distância de até 300 metros do carro.

O primeiro controlador (Steer) responsável por fazer com que o carro faça as curvas da pista tem como entrada os dados obtidos pelo sensor track. A posição do sensor que retorna o maior valor

IX COEN - Congresso de Engenharias da UFSJ Interconexão. 


\section{Seja seu próprio

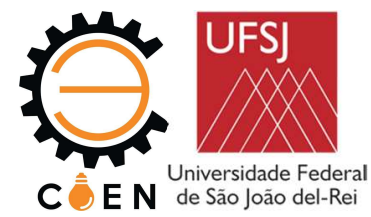

de distância da borda é usada para saber para identificarmos as curvas que o carro deve fazer. O sensor que

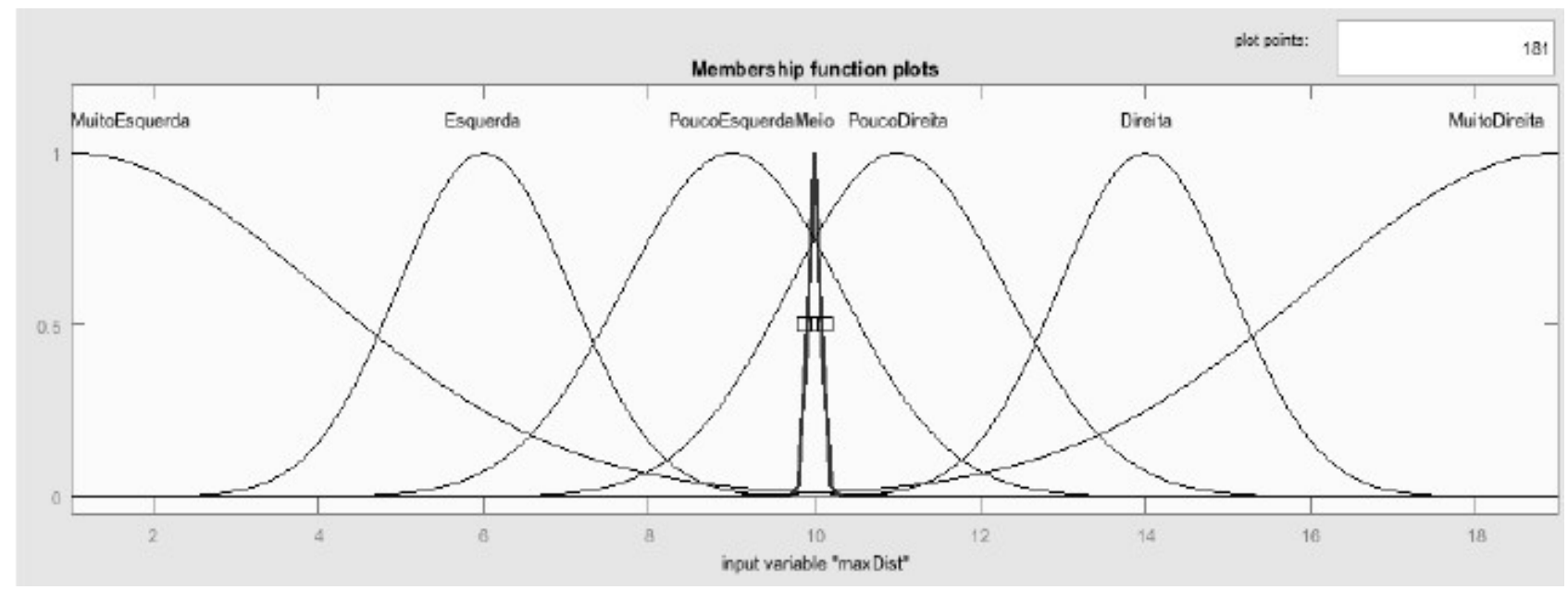

Figura 5. Funções de pertinência para a variável de entrada track para o controlador Steer.

1. If (maxDist is MuitoEsquerda) then (steer is MuitoEsquerda) (1)

2. If (maxDist is Meio) then (steer is Reto) (1)

3. If (maxDist is MuitoDireita) then (steer is MuitoDireita) (1)

4. If (maxDist is Esquerda) then (steer is Esquerda) (1)

5. If (maxDist is PoucoEsquerda) then (steer is PoucoEsquerda) (1)

6. If (maxDist is PoucoDireita) then (steer is PoucoDIreita) (1)

7. If (maxDist is Direita) then (steer is Direita) (1)

A Figura 6, mostra que a saída do controlador também é mapeada em sete funções de pertinência do tipo Gaussiana, com universo de discurso variando de -1 a 1 .

Para controlar a aceleração do veículo, é usado como informação de entrada o valor retornado pelo sensor track na posição 10 , a aceleração deve ser 1.0, ou seja, pedal totalmente acionado se a distância retornada pelo sensor for maior que 150, quando esse valor é menor que 150, usamos o controlador fuzzy "acelerador" para decidir com qual intensidade eles devem ser acionados. Após o cálculo da intensidade, se o valor retornado pelo controlador for menor que 0.09 , o pedal acionado deve ser o de freio (brake) com uma intensidade equivalente a 5 vezes o valor retornado pelo controlador. Caso o valor seja maior que 0.09 o pedal de aceleração recebe o valor retornado pelo controlador. Utiliza-se o sensor speedY para identificar se que o carro possui velocidade diferente de zero em seu eixo $\mathrm{Y}$, se houver isso significa que o veículo está derrapando na pista, caso essa afirmação seja verdadeira os valores de aceleração e freio recebem o valor 0.0 . A máquina de inferência possui apenas três regras, são elas:

If (track is PertodaCurva) then (accel is Pouco) (1)

1. If (track is EntrandoPertoCurva) then (accel is Meio) (1)

2. If (track is Reta) then (accel is Acelera) (1)

IX COEN - Congresso de Engenharias da UFSJ Interconexão. 

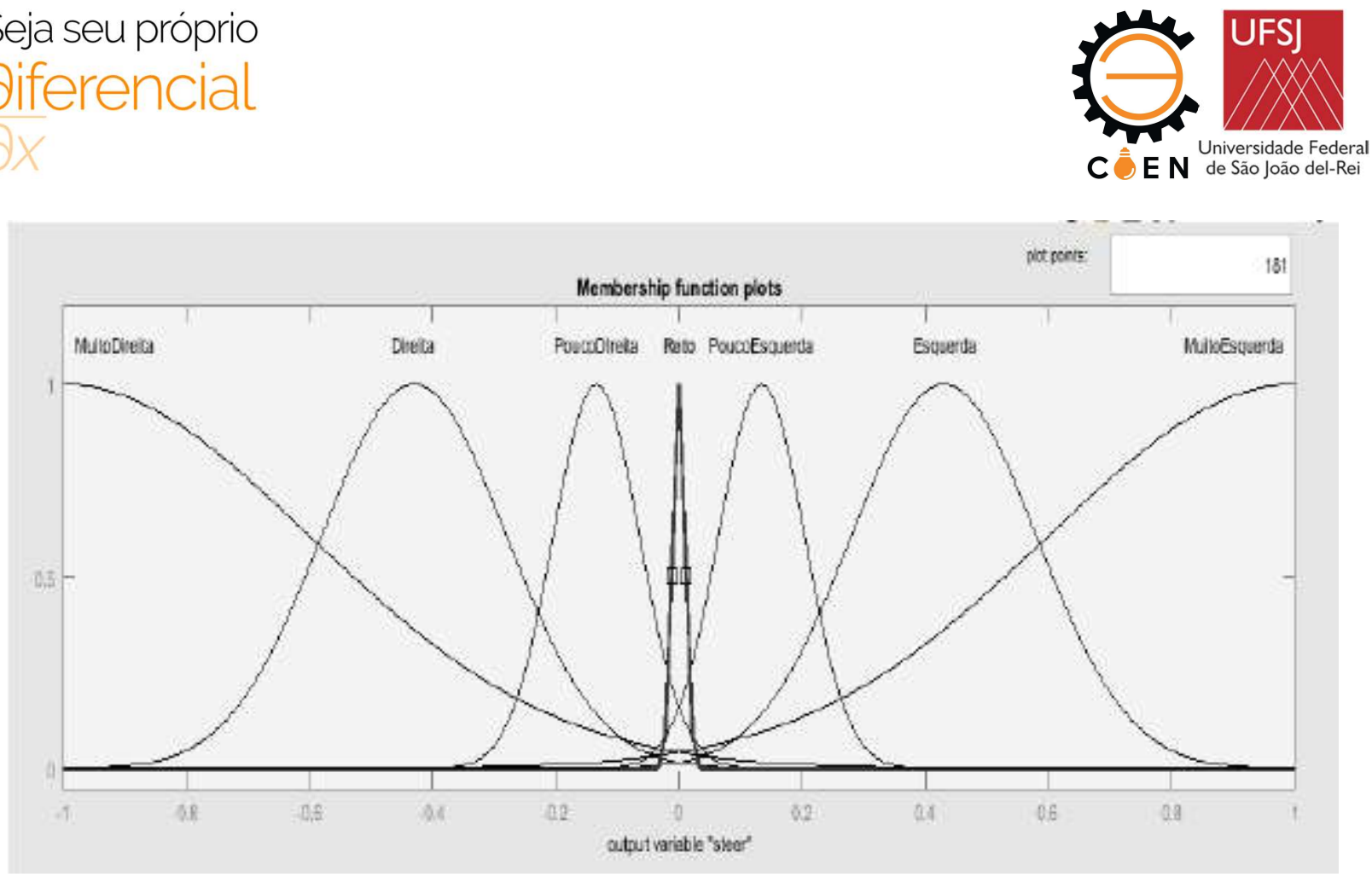

Figura 6. Funções de pertinência para a saída do sistema fuzzy Steer.

Para controlar a caixa de velocidades do veículo ( $g e a r$ ), não utiliza-se um controlador fuzzy pois o tempo de processamento desse seria muito mais elevado que uma base de regras comum, interferindo na dinâmica da simulação, além de que a saída necessária para esse comando é facilmente conseguida através do algoritmo mostrado abaixo que se mostrou muito mais eficiente. Nele é usado os sensores de velocidade speedX e track(10) para identificar se o carro está ou não batido. Caso speed $X$ seja menor que 0.1 e track(10) menor que 0.5 concluí-se que o carro está batido, então acionamos a marcha ré. $\mathrm{O}$ carro permanece em ré até que track(10) seja maior que 1.0 e o valor absoluto do sensor angle seja maior que 1.2 indicando que o carro já está na direção certa da pista para continuar a corrida. Caso o veículo não esteja batido, utiliza-se o sensor de marcha atual (sensor gear) e o sensor de velocidade (sensor rpm) para decidir qual marcha o veículo deve usar.

\section{RESULTADOS E CONCLUSÕES}

Foram feitas simulações em 3 pistas diferentes as quais são mostradas na Figura 7.

A primeira pista (OVAL TRACKs- speed-way-B) possui $3999 \mathrm{~m}$, o melhor tempo obtido foi de 1min:05s:60ms, enquanto o tempo total para completar 5 voltas foram $05 \mathrm{~min}: 50 \mathrm{~s}: 60 \mathrm{~ms}$. Para a segunda pista (DIRT TRACKs, Dirt -5), que possui 1073m obtivemos 1min:10s:34ms na melhor volta e 06min:20s:34ms para 5 voltas. A terceira pista (OVAL TRACKs, Speed-way C) teve a melhor volta completada em 55s:50ms, sendo que possui $3294 \mathrm{~m}$, as 5 voltas foram completas com 04min:57s:75ms. 
Seja seu próprio

Diferencial
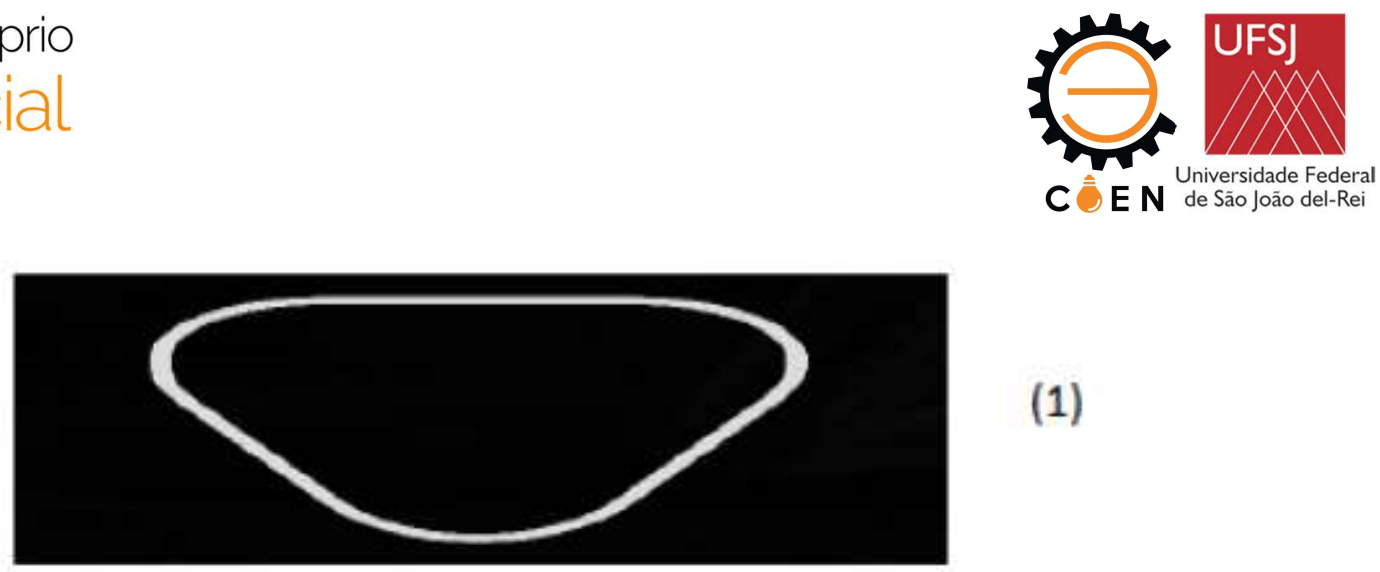

(1)

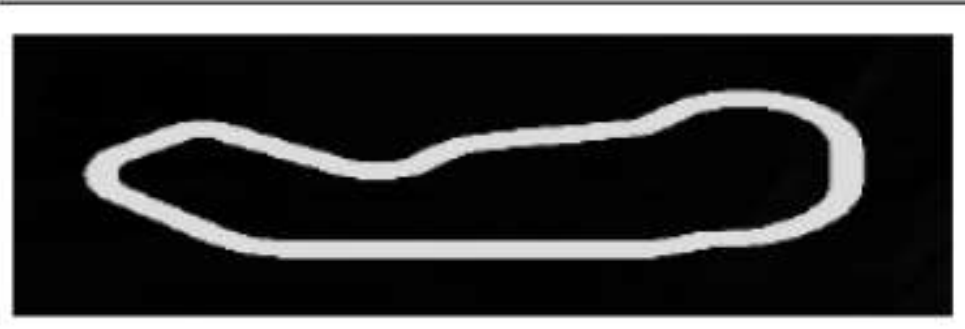

(2)

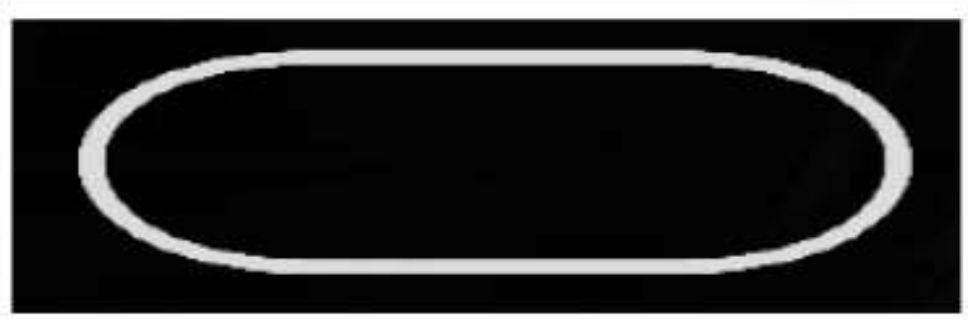

(3)

Figura 7. Pistas de teste utilizadas. (1) OVAL TRACKs (B Speed-way), (2) DIRT TRACKs (Dirt -5, (3) OVAL TRACKs (C Speed-way).

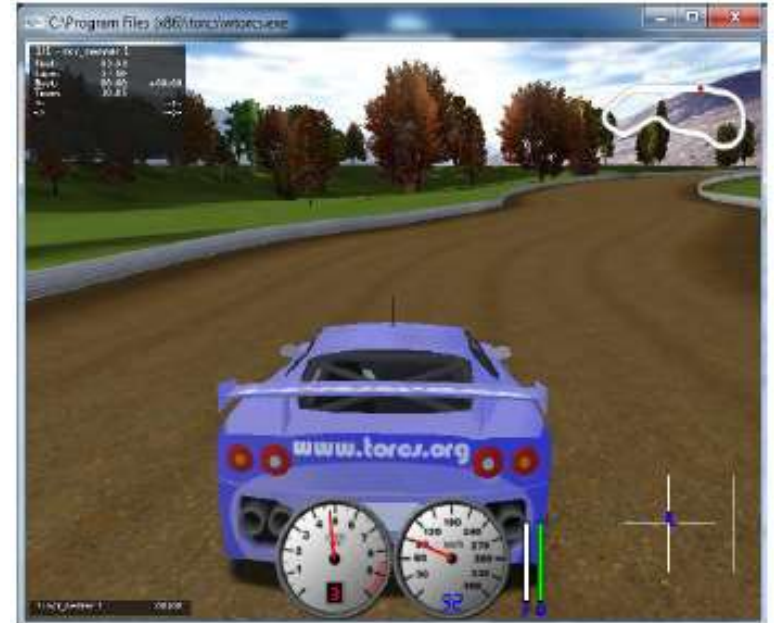

Figura 8. Pista do tipo Oval Track plataforma TORCS.

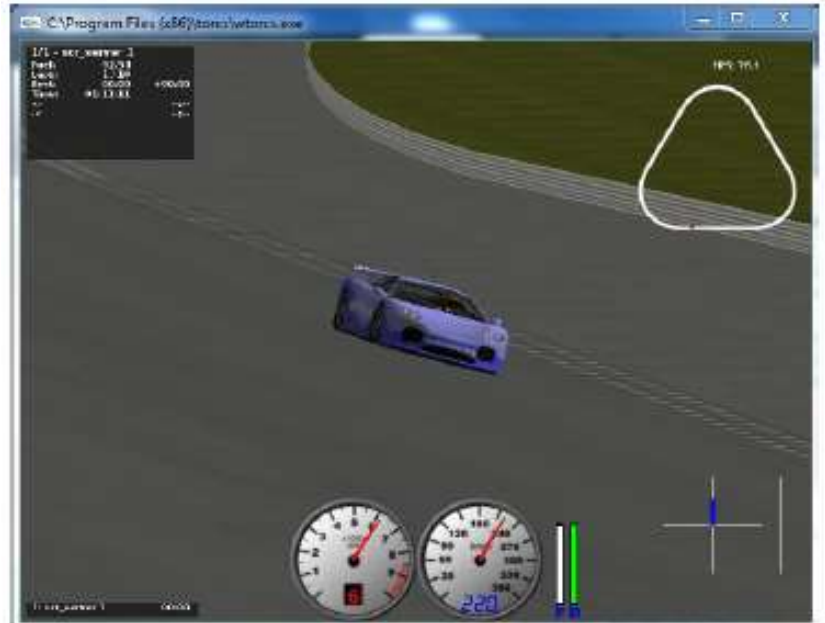

Figura 9. Controlador completando a primeira volta na pista Oval Track plataforma TORC

Como podemos ver, o veículo obteve melhor performance nas pistas do tipo OVAL TRACK, sendo a pista de tipo B (pista 1) a que apresentou maior velocidade média, isso pode ser explicado pelo

IX COEN - Congresso de Engenharias da UFSJ Interconexão. 

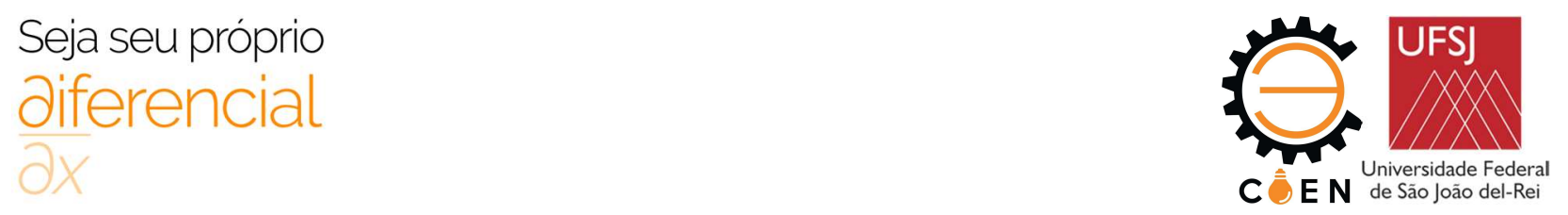

nível de dificuldade de cada pista, quantidade de curvas, tipos de curvas, aderência da pista e entre outros fatores.

Foi analisado também o esforço computacional utilizado nos controladores, como esperado é possível afirmar que com o aumento do número de variáveis e o consequente aumento no número de funções de pertinência e regras de inferência o esforço computacional também é aumentado.

Assim, conclui-se que é possível atingir o objetivo do controlador de forma rápida e bastante simples como mostrado no trabalho, porém controladores mais refinados capazes de oferecer melhores resultados, requerem maior processamento computacional. Sendo assim, tarefa de melhorar os resultados para um controlador fuzzy pode ser uma tarefa bastante complicada e onerosa.

Neste trabalho apresentamos uma breve introdução sobre o contexto de veículos autônomos e o uso de inteligência artificial na área. Também foram apresentados conceitos básicos necessários para a construção de um controlador e de sistemas fuzzy. Podemos concluir que a estrutura do controlador proposto foi eficiente para resolver o problema.

Novos sistemas fuzzy podem ser implementados para comparação de resultados.

Para otimizar os tempos e as trajetórias do veículo, técnicas de agrupamento fuzzy fundamentados na teoria de otimização não-linear, especialmente algoritmos incrementais poderiam ser implementados, bem como o uso de parâmetros e estrutura de controladores fuzzy evolutivos no tempo. A ideia é que o veículo realiza voltas cada vez mais rápidas a partir de feedback do ambiente e adaptação online de seus parâmetros. Uma abordagem neuro-fuzzy também tem se mostrado muito propícia segundo projetos já encontrados na literatura.

\section{DIREITOS AUTORAIS.}

Os autores são os únicos responsáveis pelo conteúdo das informações contidas neste artigo.

\section{REFERÊNCIAS}

CARVALHO JR, H. H. Métodos inteligentes de navegação e desvio de obstáculos. 2007. Dissertação de Mestrado - Universidade Federal de Itajubá, Itajubá-MG.

CHOSET, H. et al. Principles of Robot Motion: Theory, Algorithms and Implementation. Cambridge: MIT Press, 2005.

GAGE, Douglas W. Special Issue on Unmanned Ground Vehicles. Summer 1995, volume 13, number 3- Unmanned Systems Magazine.

LOIACONO, D., CARDAMONE, L., BUTZ, M., LANZI, P.L.: The 2011 simulated car racing championship @ cig-2011. TORCS news (2011).j Acesso em 5 nov. 2017

MAMDANI, E. H. E ASSILIAN S. (1975) An experiment in linguistic synthesis with a fuzzy logic controller. Int. J. Man-Machine Studies, vol. 7.

PEREIRA, F. L. Sistemas e veículos autônomos-aplicações na defesa. Instituto de Defesa Nacional, v. 11, 2005. Disponível em: . Acesso em: 7 jan. 2017.

PEREIRA, A.H. ; VILAÇA JR C. A.; SOARES F. G.; LEMOS M. A; DEL POÇO R. L.Um modelo fuzzy de navegação com desvio de obstáculos para o Robotino. 201?.UNESP, Sorocaba.

SECCHI, H.A. Una Introduccion a los Robots Moviles. San Juan: Universidad Nacional de San Juan, 2008.

IX COEN - Congresso de Engenharias da UFSJ Interconexão. 


\title{
FUZZY CONTROL FOR AUTONOMOUS NAVIGATION IN UNKNOWN ENVIRONMENT SUBJECT TO CHANGES
}

\author{
Larissa de Sousa Pinto ${ }^{(1)}$ (larissap@ita.br), Thaís da Costa Dias ${ }^{(2)}$ (thaisdias.engmec@hotmail.com), \\ Jorge Nei Brito ${ }^{(3)}$ (brito@ufsj.edu.br) \\ (1) Instituto Tecnológico de Aeronáutica (ITA) - Departamento de Eletrônica - Av. do Aeroporto $s \backslash n^{\circ}$ \\ (2) Universidade Federal de São João del-Rei (UFSJ) - PPMEC - Praça Frei Orlando, 170 - Centro, São João del-Rei - MG. \\ (3) Universidade Federal de São João del-Rei (UFSJ) - DEMEC - Praça Frei Orlando, 170 - Centro, São João del-Rei - MG.
}

ABSTRACT: Artificial intelligence applied to autonomous vehicle navigation has received great research attention due to a diversity of challenges still to be exploited. Research can take advantage of computational frameworks for simulation and of games. These provide test situations close to those of a real system, however considering a virtual environment free of risks, costs and of easy algorithm implementation. In the artificial intelligence context for solving navigation problems, fuzzy logic has presented a way to manage information so that human knowledge is connected to the quantitative manner that computational systems use to process data. This work proposes fundamentals and essential concepts for the implementation of a fuzzy control system for an autonomous vehicle that is able to drive several different tracks via the TORCS simulation environment. This framework consists of open code and takes into account important physical aspects of the problem such as collision, traction, aerodynamics, fuel consumption, among others. Intelligent fuzzy control is responsible to decide values for control actions, i.e., for the steer, accelerator, break and gear. The purpose is achieving faster laps.

KEYWORDS: Fuzzy Control, Autonomous Navigation, TORCS, Artificial Intelligence in Games. 